Status of the National Ignition Facility Integrated Computer Control System (ICCS) on the Path to Ignition

L. J. Lagin, R. C. Bettenhausen, G. A. Bowers, R. W. Carey, O. D. Edwards, C. M. Estes, R. D. Demaret, S. W. Ferguson, J. M. Fisher, J. C. Ho, A. P. Ludwigsen, D. G. Mathisen, C. D. Marshall, J. T. Matone, D. L. McGuigan, R. J. Sanchez, R. T. Shelton, E. A. Stout, E. A. Tekle, S. L. Townsend, P. J. Van Arsdall, E. F. Wilson

May 25, 2007

6th IAEA Technical Meeting on Control, Data Acquisition, and Remote Participation for Fusion Research Inuyama, Japan June 4, 2007 through June 8, 2007 
This document was prepared as an account of work sponsored by an agency of the United States government. Neither the United States government nor Lawrence Livermore National Security, LLC, nor any of their employees makes any warranty, expressed or implied, or assumes any legal liability or responsibility for the accuracy, completeness, or usefulness of any information, apparatus, product, or process disclosed, or represents that its use would not infringe privately owned rights. Reference herein to any specific commercial product, process, or service by trade name, trademark, manufacturer, or otherwise does not necessarily constitute or imply its endorsement, recommendation, or favoring by the United States government or Lawrence Livermore National Security, LLC. The views and opinions of authors expressed herein do not necessarily state or reflect those of the United States government or Lawrence Livermore National Security, LLC, and shall not be used for advertising or product endorsement purposes. 


\title{
STATUS OF THE NATIONAL IGNITION FACILITY INTEGRATED COMPUTER CONTROL SYSTEM (ICCS) ON THE PATH TO IGNITION*
}

\author{
L. Lagin, R. Bettenhausen, G. Bowers, R. Carey, O, Edwards, C. Estes, R. Demaret, S. Ferguson, \\ J. Fisher, J. Ho, A. Ludwigsen, D. Mathisen, C. Marshall, J. Matone, D. Mcguigan, R. Sanchez, \\ R. Shelton, E. Stout, E. Tekle, S. Townsend, P. Van Arsdall, E. Wilson
}

Lawrence Livermore National Laboratory, P.O. Box 808, Livermore, California USA

\begin{abstract}
The National Ignition Facility (NIF) at the Lawrence Livermore National Laboratory is a stadium-sized facility under construction that will contain a 192-beam, 1.8-Megajoule, 500Terawatt, ultraviolet laser system together with a 10-meter diameter target chamber with room for multiple experimental diagnostics. NIF is the world's largest and most energetic laser experimental system, providing a scientific center to study inertial confinement fusion (ICF) and matter at extreme energy densities and pressures. NIF's laser beams are designed to compress fusion targets to conditions required for thermonuclear burn, liberating more energy than required to initiate the fusion reactions. NIF is comprised of 24 independent bundles of 8 beams each using laser hardware that is modularized into more than 6,000 line replaceable units such as optical assemblies, laser amplifiers, and multi-function sensor packages containing 60,000 control and diagnostic points. NIF is operated by the large-scale Integrated Computer Control System (ICCS) in an architecture partitioned by bundle and distributed among over 800 front-end processors and 50 supervisory servers. NIF's automated control subsystems are built from a common objectoriented software framework based on CORBA distribution that deploys the software across the computer network and achieves interoperation between different languages and target architectures. A shot automation framework has been deployed during the past year to orchestrate and automate shots performed at the NIF using the ICCS. In December 2006, a full cluster of 48 beams of NIF was fired simultaneously, demonstrating that the independent bundle control system will scale to full scale of 192 beams. At present, 72 beams have been commissioned and have demonstrated 1.4-Megajoule capability of infrared light. During the next two years, the control system will be expanded in preparation for project completion in 2009 to include automation of target area systems including final optics, target positioners and diagnostics. Additional capabilities to support fusion ignition shots in a National Ignition Campaign (NIC) beginning in 2010 will include a cryogenic target system, target diagnostics, and integrated experimental shot data analysis with tools for data visualization and archiving. This talk discusses the current status of the control system implementation and discusses the plan to complete the control system on the path to ignition.
\end{abstract}

\section{INTRODUCTION}

The National Ignition Facility (NIF) is presently under construction at the Lawrence Livermore National Laboratory (LLNL). NIF is a 192-beam laser system to study inertial confinement fusion (ICF) and the physics of extreme energy densities and pressures. NIF will be the world's largest and most energetic laser experimental system, providing a scientific center to study inertial confinement fusion (ICF) and matter at extreme energy densities and pressures. NIF's laser beams are designed to compress fusion targets to conditions required for thermonuclear burn, liberating more energy than required to initiate the fusion reactions. NIF is comprised of 24 independent bundles of 8 beams each using laser hardware that is modularized into approximately 6,200 line replaceable units (LRUs) such as optical assemblies, laser amplifiers, and multi-function sensor packages containing 60,000 control and diagnostic points. When completed in 2009, NIF will be able to produce $1.8 \mathrm{MJ}, 500 \mathrm{TW}$ of ultraviolet light for target experiments. At this power and energy, NIF is expected to ignite deuterium-tritium plasmas in ICF targets.

Presently, the NIF Project is approximately 90\% complete. Construction of the laser beampath and target area infrastructure is complete, and installation of laser hardware and control systems is 
well underway. To date, approximately 3,800 LRUs have been installed and qualified. In addition, 72 beams are operational in the main laser bay. These 72 beams have produced the equivalent of $1.4 \mathrm{MJ}$ of $1.05-\mu \mathrm{m}$ light $(1 \omega)$, making it the world's most energetic infrared laser. Construction of the target area and ignition systems is also well underway, and planned to be operational for ignition experiments planned in 2010 [1].

In 2003-2004, four beams were activated to the target chamber for target experiments in a campaign called NIF early light (NEL). One of the beams was also directed to a laser precision diagnostic station (PDS). On a beam line basis, NIF demonstrated operation at all Project completion criteria and long-term functional requirements and primary criteria, as well as its capability required for ignition. NIF also performed target experiments in four experimental campaigns. Experience gained operating NIF early in the Project construction both validated the control system architecture and more clearly exposed the next level of shot automation requirements to the software development team.

The initial set of 13 target diagnostics to support laser performance characterization and target experiments were also commissioned. These included systems for imaging $\mathrm{x}$-rays generated by laser irradiation of targets, for measuring laser light scattered from the target and for characterizing $\mathrm{x}$-ray drive spectra within hohlraum targets. In addition, a velocity interferometer capable of measuring shock propagation and equation of state in materials at high pressure was commissioned. Experiments in laser-plasma interactions, laser propagation in plasmas, beam conditioning, hydrodynamic instabilities and hohlraum physics were performed.

\section{INTEGRATED COMPUTER CONTROL SYSTEM STATUS}

NIF is operated by the Integrated Computer Control System (ICCS) in an architecture partitioned by bundle and distributed among over 800 front-end processors and 50 supervisory servers. Every NIF experimental shot is a complex computerized coordination of laser equipment according to laser settings calculated by a physics model. The ICCS is a large-scale, automated system that provides reliable monitoring and control of 60,000 distributed control points comprised of electronic, optical, and mechanical devices, such as motorized mirrors and lenses, adaptive optics, energy and power sensors, video cameras, pulse power, and diagnostic instruments [2]. The precise orchestration of these parts will result in the propagation of 192, nanosecond-long bursts of light along a 1-km path. The beams must arrive within 30-ps of each other at the center of a target chamber 10 meters in diameter, and they must strike within $50-\mu \mathrm{m}$ of their assigned spot at a target measuring less than $1-\mathrm{cm}$ long.

ICCS is a hierarchically organized distributed control system that employs a scalable framework of reusable software to build uniform programs for beam control, injection laser, power conditioning, laser diagnostics, and target diagnostics [3]. The control system is comprised of two principle layers - supervisory controls performing higher operational scenarios, and lower-level front end processors (FEP) interfacing to and controlling laser hardware. ICCS employs Java, Ada95, CORBA, and object-oriented techniques to enhance the openness of the architecture and portability of the software. This common object-oriented software framework based on CORBA distribution deploys the software across the computer network and achieves interoperation between different languages and target architectures. CORBA provides transparent language binding and distribution middleware. Ada generally implements lower-level FEPs and Java is used for the production of graphical user interfaces and the integration of commercial software, particularly the Oracle database system. Higher-level supervisory software, which controls operational scenarios, is being migrated from Ada to Java.

The physical partitioning of NIF's independent bundles has been extended to the control system computer architecture. Control processes and computers are organized by bundle to achieve better parallelism, to assure predictable scaling performance, and to reduce the impact of localized failures. This is referred to as "bundle-based partitioning" and had no impact on framework or supervisory software due to the location-independent features of the CORBA distribution architecture. Bundle independence greatly simplifies the control system software because each 
bundle is operated asynchronously from the others until the final countdown, at which point all systems are synchronized and fired by the facility timing system. A large-scale, redundant Gigabit Ethernet network assures reliability, isolates the bundles from each other, and delivers predictable full scale performance in the network backbone. During the past two years, computer systems and software that were fielded for the first bundle have been installed, and activated by setting the control system configuration database to commission new bundles. A new PC-based FEP target architecture was added to leverage commercial code for firewire support of high-resolution machine vision sensors. CORBA's language and processor transparency facilitated migration of the ICCS framework to Windows XP using an alternate Ada compiler technology (AdaCore GNAT Pro). The FEP can either capture shot data or deliver compressed streaming video to the operator consoles. The network-attached camera FEP is hosted on Dell 1U servers.

An automation framework was developed and deployed to automate bundle shots [4]. NIF's 4hour shot sequence is comprised of shot lifecycle states that include reading shot goals from a physics model, aligning laser beams, setting laser parameters, configuring diagnostics, verifying critical status readiness, and conducting a final 4-minute countdown. The framework features a model-based workflow and provides scripted behaviors stored in the database that allow flexibility to modify automation instructions in the field without recompiling the software. The framework provides two major constructs to the application software: a workflow engine and a state machine. The workflow engine organizes collaboration among subsystem supervisors and coordinates transitions between shot lifecycle states. The state machine coordinates all bundle workflow engines. The automation framework will operate 24 bundles in parallel by coordinating processes distributed over the 850 processors. The new shot automation framework, deployed in April 2005, was used to perform main laser commissioning shots for the first bundle in September 2005 [Figure 1]. Flexibility of the model-based software has been demonstrated for several different shot types including setup verification, high power, and amplifier tests. In December 2006, a full cluster of 48 beams (6 bundles) of NIF was fired simultaneously, demonstrating that the independent bundle control system will operate at the full scale of 192 beams. At present, 72 beams have been commissioned and have demonstrated 1.4-Megajoule capability of infrared light.

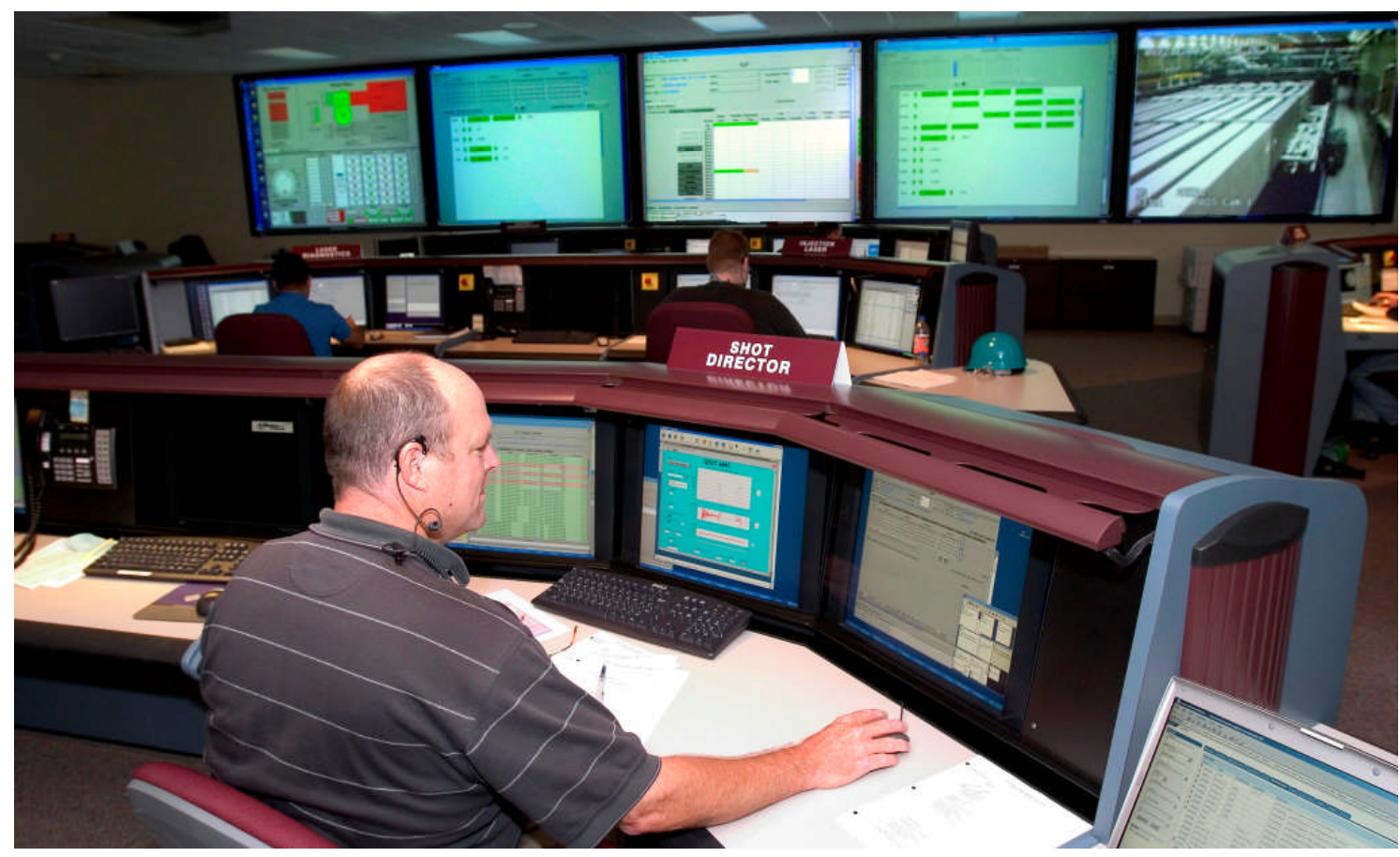

Figure 1. The NIF Shot Director oversees automated shot operations using ICCS.

Success of this large-scale project was keyed to early adoption of rigorous software engineering practices including architecture, requirements management, code design and reviews, configuration management, code unit testing, product integration, and formal verification testing [5]. Releases are formally managed by a change control board. Verification testing is performed 
in several stages. Developers integrate and test releases in a dedicated test facility, which is followed by qualification by an independent test team. Online formal tests are performed in the NIF facility through the collaborative efforts of the Commissioning and Operations, Engineering and Controls Test teams, prior to acceptance. These processes resolve most software problems early, with more than $90 \%$ of problems found prior to use for facility operations.

Quality control processes are augmented by an overarching quality assurance program featuring assessment of quality metrics and corrective actions. Quality metrics are analyzed to help determine appropriate corrective actions. For example, data indicated additional developer testing and code inspections should be used to augment intensive integration and verification testing practices already in place. Results obtained by increasing the early-phase quality controls resulted in substantially more defects being found when they are less costly to repair.

\section{PLANS TO COMPLETE ICCS}

Presently, ICCS is approximately $80 \%$ complete. A dedicated on-site FEP factory already has assembled 650 of the planned 800 FEPs with high quality. A formal acceptance process is used during FEP installation and commissioning. During the next two years, the control system will be expanded in preparation for project completion in 2009 by approximately 300 KSLOC to include automation of target area systems including final optics, target positioners and diagnostics. Controls for the final optics systems have been installed and qualified, with additional automation capabilities for optics inspection being planned for delivery by the end of 2007. Target area systems currently being qualified include systems to position targets and align beams to target chamber center, as well as chamber inspection viewing systems and systems to insert diagnostics into the target chamber. Shot automation capabilities are being developed to automate target alignment and positioning, and to automate target diagnostic setup and archiving.

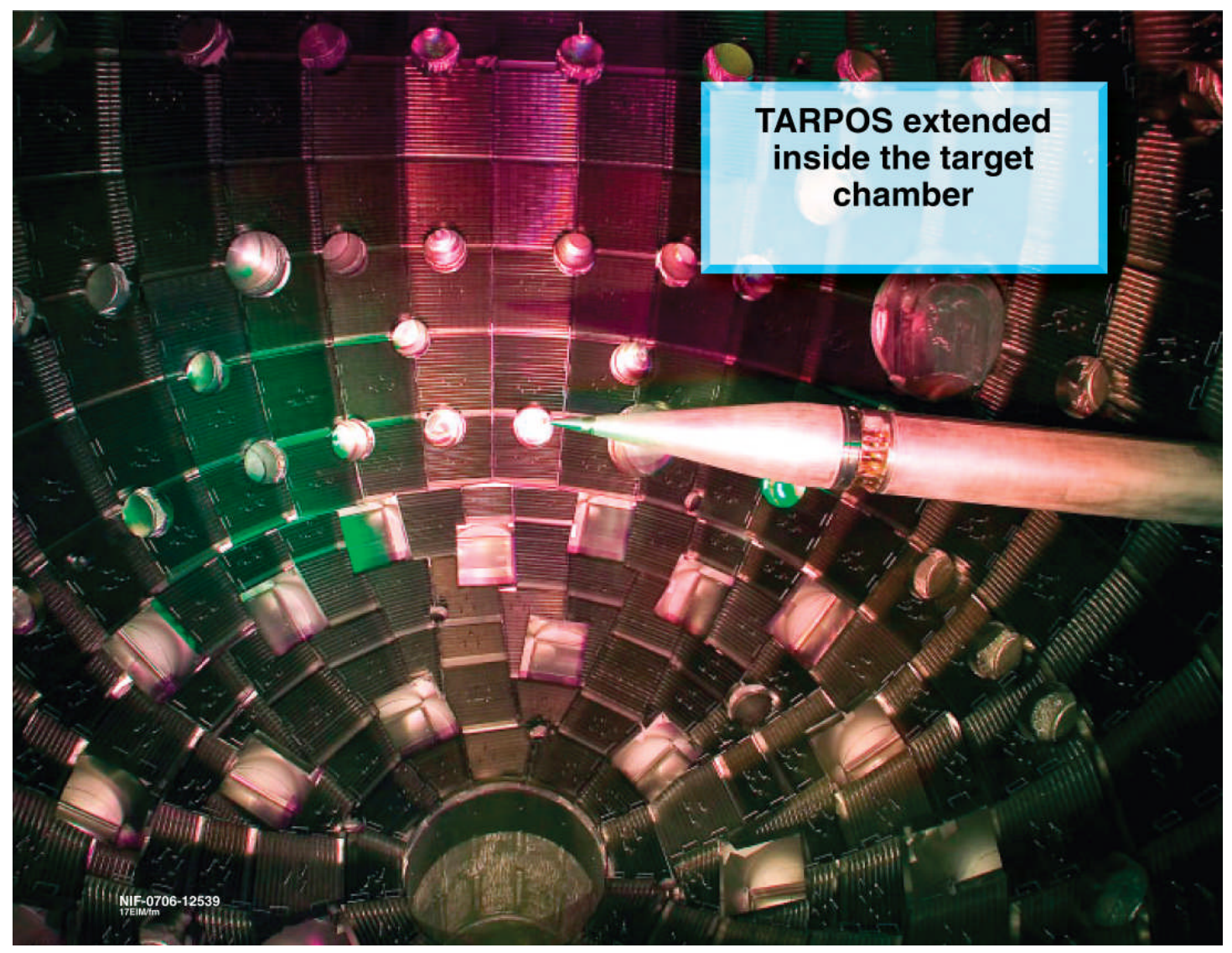

Figure 3 Target positioner extended inside the target chamber 
Additional capabilities are being developed to support fusion ignition shots planned for the National Ignition Campaign beginning in 2010. These capabilities include a cryogenic target system, more target diagnostics to diagnose ignition physics, and integrated experimental shot data analysis with tools for data visualization and archiving. The cryogenic subsystem for ignition experiments includes a cryogenic target capability consisting of a target positioner, a cryogenic shroud, target installation glove box, deuterium-tritium ice layering and characterization station, and a transport system. The target will be cooled, filled, layered and characterized next to the chamber and then inserted and aligned. [Figure 4].

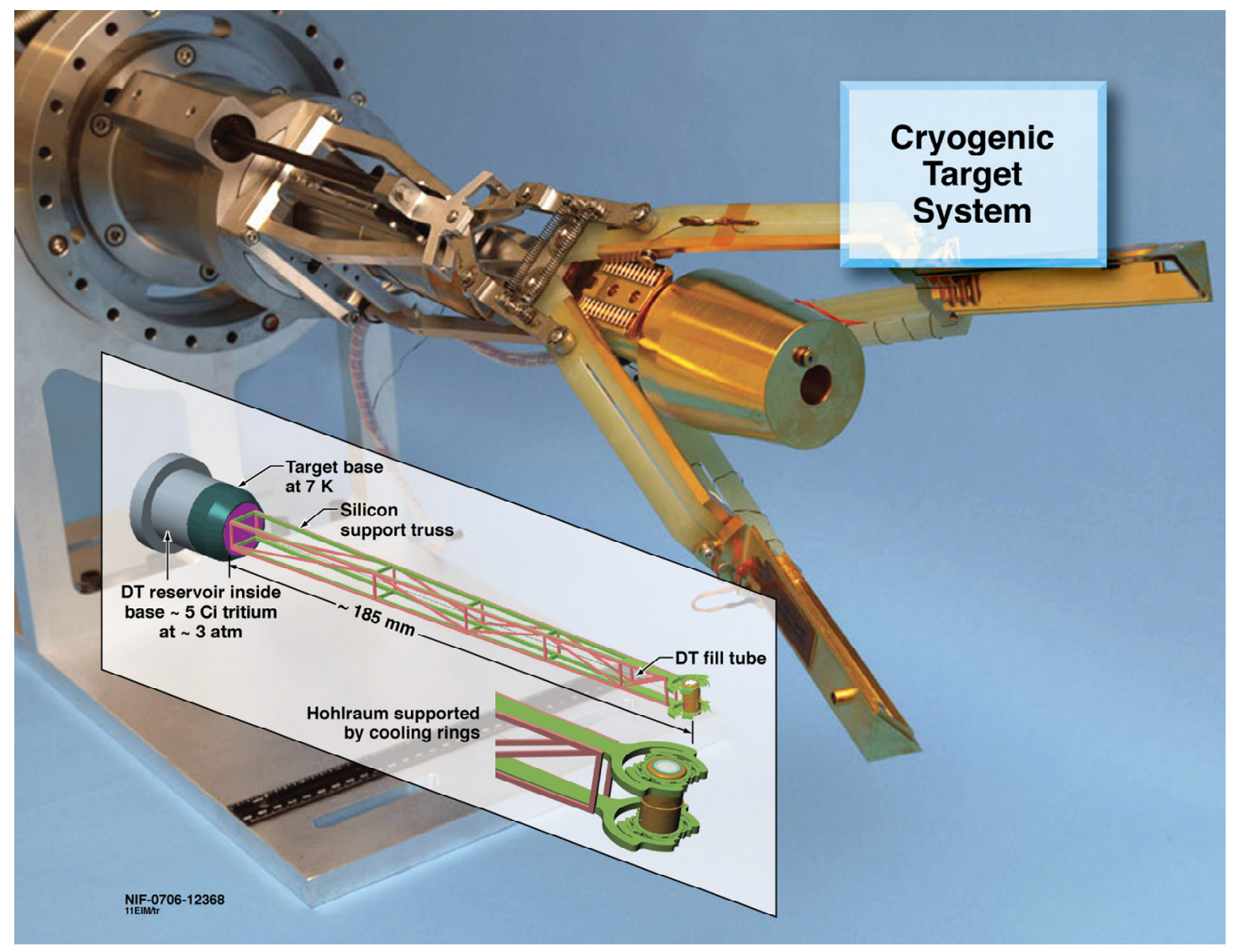

Figure 4. Cryogenic target positioner

Over 30 types of diagnostic systems are planned for the National Ignition Campaign. Efficient tools for controlling a diverse suite of target diagnostic instruments have led to the development of a instrument-based controls (IBC) architecture in Java [6]. This architecture facilitates the integration of components and software developed by collaborators from other institutions, and minimizes development, testing and maintenance costs. The IBC architecture uses a low-cost $\mathrm{PC} 104$ processor to control each device (e.g., a digitizer). The full diagnostic control system is composed within the supervisory software by aggregating the set of network-attached controllers. This approach simplifies the embedded software, improves reliability and provides easy re-use of devices for diagnostics.

Shots for the National Ignition Campaign will leverage the data-driven shot automation framework which supports database-driven models. Types of shots to be supported include laser performance qualification, target physics verification, non-cryogenic implosion and cryogenic ignition implosion shots. Data systems are being developed to support efficient storage, analysis and visualization of experimental results. NIF shot data will be managed in a large-scale, centralized data repository with access controls, back-up and retrieval support, configuration management, and interfacing tools to retrieve data [7]. Production analysis codes will be integrated into a model-based shot analysis framework for NIC experiments to provide integrated 
physics codes that automatically run during and after a shot. Online and offline data visualization and analysis tools will allow users to view and analyze NIF data.

After the NIF Project is completed in 2009, the goal is to begin ignition experiments in 2010. An integrated National Ignition Campaign plan developed to meet this goal is underway. The plan integrates target physics and NIF operations tasks into a multiyear effort with initial ignition experiments beginning in 2010 .

\section{SUMMARY}

The ICCS framework supports experimental physics control systems and accommodates the complexities of large-scale distribution. The software architecture and CORBA middleware allow easy reconfiguration of the computer control system to optimize performance. Bundle-based controls alleviated concerns that the computer and software architecture could scale successfully as NIF was built out. Model-driven shot automation software successfully reduced efforts required of system operators, achieved the required shot rate, and afforded operational flexibility.

ICCS is operational $24 \times 7$ and is actively used 3-shifts a day, 5 days per week. Many parallel tasks are currently exercising the control system. Rigorous quality controls assure delivery of reliable hardware and software to the facility. Automated multi-bundle shots allowed the facility to fire shots at increased efficiency, while protecting the laser equipment from potential mistakes. Sixbundle (48 beamlines) shots have demonstrated capability to scale to full NIF. Shot automation of target area systems is underway, including control of final optics, target positioners and diagnostic systems. Control systems are being developed to support ignition experiments in 2010. These systems include a cryogenic target system, target diagnostics, and integrated experimental shot data analysis with tools for data visualization and archiving.

\section{REFERENCES}

[1] E. Moses, "Plans for Ignition Experiments on the National Ignition Facility", 6th IAEA Technical Meeting on Control, Data Acquisition and Remote Participation for Fusion Research", June 2007, Inuyama, Japan.

[2] P. VanArsdall, et al, "Status of the National Ignition Facility and Control System", ICALEPCS 2005, Geneva, Switzerland, October 2005.

[3] E. Stout, et al, "CORBA-Based Distributed Software Framework for the NIF Integrated Computer Control System", $6^{\text {th }}$ IAEA Technical Meeting on Controls for Fusion Research, June 2007, Inuyama, Japan.

[4] L. Lagin, et al, "Shot Automation for the National Ignition Facility (NIF)", ICALEPCS'2005, Geneva, Switzerland, October 2005.

[5] D. Casavant, et al., "Testing and Quality Assurance of the Control System during NIF Commissioning", ICALEPCS'2003, Gyeongjiu, Korea, October 2003, p. 16.

[6] D. O’Brien, et. al., “Target Diagnostic Instrument-Based Controls Framework for the National Ignition Facility (NIF), $6^{\text {th }}$ IAEA Technical Meeting on Controls for Fusion Research, June 2007, Inuyama, Japan.

[7] T. Frazier, et. al, "The National Ignition Facility Data Archive", ), $6{ }^{\text {th }}$ IAEA Technical Meeting on Controls for Fusion Research, June 2007, Inuyama, Japan.

*This work was performed under the auspices of the U.S. Department of Energy by the University of California, Lawrence Livermore National Laboratory under contract No. W-7405-ENG-48. 